\title{
Breaking the Limits - Multichromosomal Structure of an Early Eudicot Pulsatilla Patens Mitogenome Reveals Extensive RNA-editing, Longest Repeats and Chloroplast Derived Regions Among Sequenced Land Plant Mitogenomes
}

Kamil Szandar ( $\boldsymbol{\nabla}$ kamil.szandar@uwm.edu.pl )

University of Warmia and Mazury in Olsztyn

Katarzyna Krawczyk

University of Warmia and Mazury in Olsztyn

Kamil Myszczyński

Medical University of Gdańsk

Monika Ślipiko

University of Warmia and Mazury in Olsztyn

Jakub Sawicki

University of Warmia and Mazury in Olsztyn

Monika Szczecińska

University of Warmia and Mazury in Olsztyn

\section{Research Article}

Keywords: Pulsatilla, mitogenome, RNA-editing, Ranunculales, intracellular transfer, phylogeny

Posted Date: November 15th, 2021

DOI: https://doi.org/10.21203/rs.3.rs-955711/v1

License: (9) (1) This work is licensed under a Creative Commons Attribution 4.0 International License. Read Full License

Version of Record: A version of this preprint was published at BMC Plant Biology on March 9th, 2022. See the published version at https://doi.org/10.1186/s12870-022-03492-1. 


\section{Abstract}

Background: The mitogenomes of vascular plants are one of the most structurally diverse molecules. In the present study we characterize mitogenome of a rare and endangered species Pulsatilla patens. We investigated the gene content and its RNA editing potential, repeats distribution and plastid derived sequences.

Results: The mitogenome structure of early divergent eudicot, endangered Pulsatilla patens does not support the master chromosome hypothesis, revealing the presence of three linear chromosomes of total length $986613 \mathrm{bp}$. The molecules are shaped by the presence of extremely long, exceeding $87 \mathrm{kbp}$, repeats and multiple chloroplast derived regions including nearly complete inverted repeat. Since the plastid IR content of Ranunculales is very characteristic, the incorporation into mitogenome could be explained rather by intracellular transfer than mitochondrial HGT. The mitogenome contains an almost complete set of genes known from other vascular plants with exception of rps10 and sdh3, the latter being present but pseudogenised. Analysis of long ORFs enabled the identification of genes which are rarely present in plant mitogenomes, including RNA and DNA polymerases, albeit their presence even at species level is variable. Mitochondrial transcripts of P. patens were edited with a high frequency, exceeding the level known in other analyzed angiosperms, despite strict qualification criteria of editing event's count and analysis of generally less frequently edited leaf transcriptome. The total number of edited sites was 902 and nad4 was identified as the most edited gene with $65 \mathrm{C}$ to $\mathrm{U}$ changes. Non-canonical, reverse $\mathrm{U}$ to $\mathrm{C}$ editing was not detected. Comparative analyses of mitochondrial genes of three Pulsatilla species revealed a level of variation comparable to chloroplast CDS dataset and much higher infrageneric differentiation than in other known angiosperm genera. The variation found in CDS of mitochondrial genes is comparable to values found among Pulsatilla plastomes. Despite a complicated mitogenome structure, 14 single copy regions not splitted by repeats or MTPT of $329 \mathrm{kbp}$ revealed potential for phylogenetic, phylogeographic and population genetics studies by revealing intra- and interspecific collinearity.

Conclusions: This studies provides valuable new information about mitochondrial genome of early divergent eudicots, Pulsatilla patens, revealed multi-chromosomal structure and shed new light on mitogenomics of early eudicots.

\section{Background}

The mitogenomes of vascular plants are one of the most structurally diverse molecules despite generally stable gene content. After the divergence of evolutionary lineages of bryophytes and early vascular plants the mitogenomes of the latter started to expand their intergenic regions [1]. Most of the structural variation in flowering plant mitogenomes are related to the presence of large repeats which enable homologous recombinations. In addition to the large, frequently recombining repeats, there are often smaller repeated sequences in the size lower than $1 \mathrm{kbp}$ [2].The frequency of the recombination appears to be positively correlated with the length of repeats [3].

The size variation of angiosperm mitogenomes can be spectacular even between closely related taxa. Beside duplication of large parts of mitogenomes, the size expansion can be achieved by uptaking foreign sequences from plastid and nucleus or even extrinsic mitochondrial DNA via horizontal transfer [4]. The plastid derived regions contribute 1 up to $10 \%$ of the mitochondrial genomes size in vascular plants, however the majority of transferred genes were non-functional with a few exceptions of tRNA genes [5]. 
Unlike in non-flowering plants $[1,6,7]$, the mitochondrial genome is present under different forms, not only circular one [8].

In recent years several comparative analyses between closely related species were conducted, often revealing variation in structure and gene content $[6,8,9]$, but the intraspecific variation was not the subject of many studies [10]. Due to mostly maternal inheritance mitogenomes may be an important source of evolutionary information, providing new insights into plant phylogeography and population genetics. However, frequent changes of chondriome structures and assumed lower evolutionary rate than plastomes do not make mitochondrial genomes a first choice for studies on phylogeny and phylogeography, with exception of early land plant, bryophytes, which mitogenomes are rather evolutionarily stable $[1,6,7]$ with rare exceptions [11].

Modern technologies including long-read sequencing enabled obtaining sequences of many mitogenomes, but most of them belong to model or economically important and well studied species [8, 12]. Both methods of longread sequencing require high quality, high molecular weight DNA, which is sometimes difficult to obtain for species of limited tissue availability. In this study we employed PacBio and ONT sequencing technologies combined with DNA and RNA short-read sequencing to characterize mitogenome of a rare and endangered species Pulsatilla patens, belonging to Ranunculales order, which is basal for flowering plants although still poorly explored in terms of mitogenomics. In the present study we investigated the gene content and its RNA editing potential, repeats distribution and plastid derived sequences. We also tried to answer the question if mtDNA can be a source of evolutionary information in phylogeography and conservation of endangered species.

Assuming the structural dynamics of mitogenomes, the application of complete genomic sequence in microevolutionary studies of vascular plants may be problematic due to relatively difficult assembly as well as a proper interpretation of recombination events. However, in this study we tried to identify and validate single copy mitogenomic regions, which can serve as a potential resource for population scale studies using mtDNA information.

\section{Methods}

\section{Plant Material and nucleic acids extraction}

Plant material used in this study was collected from Polish population of Pulsatilla patens (P13 - Rudne, Poland; $53^{\circ} 23^{\prime} \mathrm{N}, 21^{\circ} 35^{\prime} \mathrm{E}$, Figure 4) during previous population studies [34]. Formal identification of the plant material was performed by Monika Szczecińska.

All plant material has been deposited in Herbarium of Department of Botany and Nature Protection in Olsztyn (OLS) with specimen label: Pulsatilla patens P13 - Rudne.

The DNA and RNA was isolated from the leaves of the same individual. The mature leaves of the healthy plant during its flowering stage were collected and used for total RNA extraction immediately after collection using modified phenol/SDS method for plant RNA preparation [73]. Adequate RNA quality and quantity of RNA samples was ensured by Bioanalyzer (Agilent) analysis. The RNA integrity value was measured using 
Bioanalyzer 2100 (Agilent Technologies, Santa Clara, California, USA). The purified total RNA was used for sequencing library preparation.

Total genomic DNA extracted from fresh tissue immediately after collection. Ca $1 \mathrm{~cm} 2$ of cleaned leaf tissue was ground with silica beads in a MiniBead-Beater homogenizer for $50 \mathrm{~s}$ and subsequently processed following CTAB protocol [74].

DNA quantity was estimated using the Qubit fluorometer and Qubit ${ }^{\mathrm{TM}}$ dsDNA BR Assay Kit (Invitrogen, Carsbad, NM, USA). DNA quality was checked by electrophoresis in $0.5 \%$ agarose gel stained with Euryx Simple Safe (Eurx, Gdańsk, Poland). The extracted DNA prior to long-read sequencing was carefully examined and additionally cleaned using Genomic DNA Clean and Concentrator kit (Zymo, Irvine, USA).

\section{Genomic DNA Illumina sequencing}

The genomic libraries for short-read sequencing were constructed with TruSeq DNA kit (Illumina, San Diego, CA, USA) and was sequenced using HiSeqX (Illumina) to generate 150 bp paired-end reads at Macrogen Inc. (Seoul, Korea) with 350 bp insert size between paired-ends.

\section{Nanopore sequencing}

The long-read libraries were constructed using Ligation Sequencing Kit SQK-LSK109 (Oxford Nanopore Technologies) and NEBNext ${ }^{\circledR}$ Companion Module for Oxford Nanopore Technologies ${ }^{\circledR}$ Ligation Sequencing (New England Biolabs) according to manufacturer's protocol and sequenced using MinION MK1B portable device (ONT) and R.9.4 Flow Cell (ONT). The Flow Cell was prepared for sequencing with Flow Cell Priming Kit EXP-FLP002 (ONT). Sequence reads were basecalled using high-accuracy guppy basecalling on MinKNOW platform.

\section{PacBio sequencing}

The sample was prepared according to a guide for preparing SMRTbell template for sequencing on the PacBio Sequel System. The library preparation and sequencing were done by Macrogen Inc. (Seoul, Korea).

\section{Reads polishing}

In order to use sequencing reads of the best quality the nanopore reads were polished using hybrid read error correction method. First, the Burrows-Wheeler Transform (BWT) of the short-read Illumina dataset was constructed using ropeBWT2 [75]. Next, FMLRC [76] was used to build FM-index and correct errors occurring within nanopore reads.

\section{RNA sequencing}

Total RNA extraction was performed immediately after collection using modified phenol/SDS method for plant RNA preparation [73]. Adequate RNA quality and quantity of RNA samples was ensured by Bioanalyzer (Agilent) analysis. The RNA integrity value was measured using Bioanalyzer 2100 (Agilent Technologies, Santa Clara, California, USA). The purified total RNA was used for sequencing library preparation. The extracted RNA was used for library construction using Truseq RNA kit with Ribo-Zero option (Illumina) and sequenced using Illumina NovaSeq 6000 platform by Macrogen Inc. (Seoul, Korea).

\section{Mitogenome assembly and annotation}


To avoid extensions of chloroplast genome sequences, the complete plastid genomes were assembled using NOVOplasty v2.8. [77] with previously published Pulsatilla patens plastome as reference [32]. The unmapped reads generated from three platforms were used for contig assembly using SPAdes hybrid assembler. The obtained contigs were imported into Geneious Prime to identify contig containing mitochondrial genes based on available plant mitogenomic resources in GenBank. Initial repeat analysis revealed presence of multiple repeats in 100-1000 bp range and to avoid misassembly the nanopore and PacBio reads shorter than $2 \mathrm{kbp}$ were removed from further analyses. The sequences containing mitochondrial genes were extended by mapping using Geneious mapper with five iterations with minimal overlap of $3000 \mathrm{bp}$ and overlap identity of $95 \%$. This approach enabled assembly of six scaffolds containing all known mitochondrial genes. These sequences were flanked by large 8- $87 \mathrm{~kb}$ repeats. Careful examination of nanopore reads enabled the proper orientation of three flanking regions reducing the number of scaffolds to three, which can not be assembled together due to opposite orientation of flanking repeats. The assembly was verified by mapping of PE reads with min overlap of 140 bp and identity of $99 \%$ which revealed ca $600 x$ coverage of single copy regions and proportionally higher at repeats and plastid derived regions.

Mitochondrial protein coding genes were annotated using MITOFY web-based software [28]. The congruence with ORFs predicted by Geneious software (with 300 bp minimal length) were manually checked. Exonic-intronic boundaries were corrected using RNA-seq library reads. The ORFs identified within the intergenic spacer and longer than $300 \mathrm{bp}$ length were blasted against the GenBank database using BLASTX. The rRNA and tRNA genes were identified using RNAmmer 1.2 [78] and tRNAscan-SE version 1.21 [79], respectively. The repeats were identified using RepeatFinder plugin for Geneious suite with minimum length set as $500 \mathrm{bp}$ and up to $15 \%$ mismatches between repeats. The transposable elements were detected and classified using the LTR_Retriver package [80].

\section{Identification and confirmation of RNA editing sites}

In order to predict C-to-U and U-to-C RNA editing sites, the PREPACT 3.12.0 (Universität Bonn, Bonn, Germany) [65] tool was used with the BLASTX mode and 0.001 e-value cut-off.

The RNA-seq reads obtained from the same individuals as DNA were used to confirm predicted RNA editing sites. The transcriptome and genome datasets were compared using RES-Scanner with default settings [81]. The editing frequency were calculated using previously published approach [63].

\section{Phylogenetic analyses}

The phylogenetic relationships were investigated using three datasets. The main dataset contains 29 proteincoding genes present in the most known angiosperm mitogenomes including: atp1, atp4, atp6, atp8-9, ccmB, $c c m \mathrm{C}, c c m F C, c c m F N, c o b, c o x 1-3$, matR, mttB, nad1-7, nad9, rp/2, rp/5, rp/10, rp/16, rps1-4, rps8, rps11-14, rps19 and $s d h 4$. Next two datasets including RNA and DNA polymerase genes which were found in $P$. patens and other species mitogenomes.

Extracted protein-coding genes were aligned using MAFFT [82] and trees were calculated using IQ-Tree [83] under the model automatically selected by IQ-TREE ('Auto' option in IQ-TREE) for 5000 ultrafast [84] bootstraps, as well as the Shimodaira-Hasegawa-like approximate likelihood-ratio test [85]. Optimal evolutionary models for each gene were selected on the basis of BIC criterion calculated using Modeltest GTR+F+|+G4 [86, 87]. 


\section{Variation detection}

The sequences of $P$. alpina and $P$. pratensis mitochondrial genes were obtained by mapping short reads to $P$. patens mitochondrial genes dataset extracted from assembled genome.

The SNPs were detected using Geneious Prime 2019 software (Biomatters, Auckland, New Zealand) with options: minimum coverage set to 20 and minimum variant frequency set to 0.8 . The pi diversity values were obtained using Tassel 5.2.60 software [88].

\section{Results \& Discussion}

Sequencing results

The initial assembly strategy assumed using only PE and PacBio long reads, which should overcome issues with plastid derived regions as well as most of long repeats which form the most sequenced plant mitogenomes not exceeding 1-10 kbp range [2]. However, obtained PacBio sequencing results (2 Gbp) revealed mitochondrial reads up to $2 \mathrm{kbp}$ and coverage ranged from 4-10x, which only resolved part of issues related with long repeats and plastid derived regions. Better results were obtained with the nanopore sequencing, where 24-hour runs enabled the obtaining over $8 \mathrm{Gbp}$ of data. This resulted in 60x coverage of single copy mitochondrial regions and enabled proper orientation of repeat flanking contigs.

Mapping PE read onto assemble mitogenome revealed mean 531x coverage for single copy regions and relevelant multiply for repeat regions (up to $5000 \mathrm{x}$ coverage).

Mitogenome structure

Obtained results do not support the master chromosome hypothesis either in circular or linear form. Hybrid assembly using ONT and Illumina reads enabled to obtain three mitochondrial contigs (named chromosomes chMt1-chMt3) flanking by overlapping repeats A - D, which were also found inside chromosomes chMt1 and chMt2 (Figure 1).

The main role in shaping the structure of Pulsatilla mitogenome are up to $87 \mathrm{kbp}$ - long repeats (Figure 1). The longest of them, Repeat $\mathrm{D}$ is located at 5' end of chromosome chMt1 and 3' end of chromosome chMt2. This repeat also contains remaining long repeats including almost $39 \mathrm{kbp}$ - long Repeat C, $13 \mathrm{kbp}$ Repeat B and 8 kbplong Repat A (Figure 2).

The Repeat $C$ is flanking the $5^{\prime}$ end of chromosome chM3t and appears also inside chromosome chMt1. The $5^{\prime}$ end of chromosome chMt2 is flanked by Repeat B, which is also an internal part of chromosome chMt1, while remaining ends of chromosomes are flanked by Repeat $A$.

In the case of Pulsatilla patens the linearity of chromosomes chMt1 and chMt2, which contain large repeats A, B and $\mathrm{C}$ was supported only by six and eleven nanopore reads respectively, but junctions were positively validated by long-range PCR amplification. However designed PCR primers to single copy regions closest to flanking repeats did not amplify any detectable products, which may support circular master chromosome hypothesis. The fact that single circular molecule is an oversimplified representation of the plant mitochondrial genomes and that they rather exist in vivo as a mix of circular, linear and branched forms is well documented $[8,13,14]$. 
However, a circular structure can usually be observed at the level of sequence assembly due to the presence of multiple repeats. This is not the case for $P$. patens where internal repeats are also present but their location does not allow to reconstitute the master circle chromosome. It's also indirectly supported by unequal short and long reads coverage distribution along the main flanking repeat. The linear of $P$. patens mitochondrial chromosomes is also supported by features usually found in other species with linear single or multichromosomal mitochondrial genome: a terminal inverted repeat and the genes of RNA and DNA polymerases [15]. The multichromosomal, linear mitogenomes were not previously reported in early eudicots, since Aconitum, Anemone and Nelumbo support master circular chromosome hypothesis [16, 17].

Multichromosomal architecture of the mitochondrial genome was previously reported in several tracheophytes species and can form circular, linear or branched molecules. The diversification of mitogenome structures appear in the earliest tracheophyte lineages, while the evolutionarily older land plants, bryophytes exhibit single circular mitogenome molecules [18]. Starting with lycophytes, where single circular mitochondrial chromosome were confirmed only for Huperzia [19, 20, 21], non-circular and multichromosomal mitogenomes are widely distributed among different fern, gymnosperm and angiosperm lineages. Two circular mitochondrial chromosomes were found in early divergent fern Psilotum nudum, while in Ophioglossum californicum supports master circle chromosome hypothesis [22]. The sequenced gymnosperm mitogenomes of genera Cycas, Ginkgo, Welwitschia and Taxus could be also assembled into single circular molecule [22, 23, 24], but the larger, over 5 Mbp mitogenomes of Picea species are described as multichromosomal and linear [25, 26]. The mitogenomes of angiosperms are structurally variable at genus or even species level. The largest and smallest known angiosperm mitogenomes belong to the species of Silene, which exist in single or multichromosomal, but usually circular forms [10]. Single and mulichromosomal circular mitogenomes were reported for variety of Allium cepa [27], and linear, branched or circular mitochondrial molecules were found in single individual of Lactuca sativa [8]. Out of three mitochondrial chromosomes of Solanum tuberosum two could be assembled as circular, but the third exist in the linear form [12].

The presence of master circular mitogenome structure can not be completely excluded. The assembly of Chrysantheum nakingense mitogenome using similar approach confirmed the master chromosome structure relying only on 4 nanopore reads, but modules were not flanked by long inverted repeats [14], as it was in the case of P. patens.

Gene content

Annotation of Pulsatilla patens mitogenome enables the identification of a set of genes (rRNA, tRNA and CDS) that is typical for angiosperms (Table 1). According to classification of [3] sequenced mitogenomes contain all "core" genes (atp1, atp4, atp6, atp8 atp9, ccmB, ccmC, cmmFc, ccmFn, cob, cox1-3, matR, mttB, nad1-7, nad9) and most of genes defined as "variable" (rp/1, rp/5, rp/10, rp/16, rps1-4, rps7, rps11-14, rps19, sdh4). These genes were also found in the three remaining Pulsatilla species including $P$. alpina, $P$. pratensis and $P$. vernalis. From the "variable" gene list only rps10 and sdh3 were not found in the Pulsatilla mitogenome. The fragments of sdh3 including exons 1 and 2 were found at chromosome chMt3, but they contain two internal stop codons and cover ca. $40 \%$ of functional genes. Both genes were not detected in RNA-seq analysis, so they were unlikely to be transferred to the nucleus. The presence of this gene pair (rps10 and sdh3) in plant mitogenomes seems to be correlated, since out of 18 cases of rps10 or sdh3 absence, in 15 cases both are missing [3]. Despite using deep 
sequenced libraries enabling nuclear genome coverage above 40X, the presence of these genes was not confirmed even in nuclear genomes.

The repeat units in the $P$. patens mitochondrion contain three protein coding genes: nad3, nad5 (exons 1-3) and rps12. All three genes are located in the Repeat $\mathrm{A}$, over $48 \mathrm{kbp}$ long duplicated region, resulting in two identical copies in the mitogenome. The different copy of rps12 is located in a single copy region with pairwise identity $81.9 \%$. The RNA-seq analysis confirmed the expression of both rps12 variants. These genes are also duplicated or even triplicated in few known mitogenomes including Cycas taitungensis (AP009381), Oryza minuta (NC_029816) and Vitis vinifera (NC_012119). In the case of the mitogenome of Daucus carota (NC_017855), the rps12 gene is triplicated, but opposite to Pulsatilla mitogenome, all three copies are identical. The partial duplication of nad5 gene was also described in mitogenomes of Beta vulgaris (BA000024), Cynanchum auriculatum (MH410146), Rhazya stricta (KJ485850) and Tamarindus indica (MN017227).

Pulsatilla patens mitogenome consists of two large ORFs encoded polymerases, which were recently reported in plant mitogenomes $[8,14]$. The 2,850 bp long Pp_DNA_pol_B (encoding DNA polymerase) gene and 2,154 bp Pp_RNA_pol (encoding DNA-dependent RNA polymerase) are located at the chromosome chMt1.

The length of $P p \_D N A-p o l \_B$ gene is similar in size to previously reported $A c \_D N A \_p o l \_B$ gene $(2,814 \mathrm{bp})$ found in Actinidia chinensis but the homolog of $P p_{-} R N A \_p o /$ gene of this species is remarkably shorter counting 1,743 bp.

Mining available angiosperm mitogenomes towards polymerases encoding genes revealed their presence in over 50 accessions in the case DNA polymerase and 18 accessions in the case of RNA polymerase assuming blastx identity threshold above $>50 \%$.

Both genes were reported from several mitogenomes, but the expression of them remained unconfirmed in most of the studies. Angiosperm mitochondrial genomes contain DNA derived from exons of the nuclear genome [28, $29,30]$, but the examples of expression are rather rare. The expression of nuclear derived gene arf17 was confirmed in mitogenomes of Arabidopsis [31], but it's rather a rare exception. The RNA-Seq analysis confirmed the presence of mRNA of polymerase coding genes in all three analysed samples. However, since availability of nuclear genomic resources of $P$. patens is limited to NOR regions [32, 33], the nuclear origin of these transcripts can not be ruled out.

The presence of polymerase coding genes seems to be limited to $P$. patens. Both genes are absent in the three other Pulsatilla species, including $P$. alpina, $P$. pratensis and $P$. vernalis as well as in more distant species of Ranunculales, Anemone maxima and Aconitum kusnezoffii. Moreover, among eight analysed specimens of $P$. patens, the presence of polymerase like genes was not confirmed in individuals from populations STR6 and a115. The frequency of intraspecific presence polymerase-like genes was not analysed in previous studies, but the lack of these genes in samples from southern populations [34] could indicate its recent transfer from nucleus.

Table 1. Gene contents, localization and RNA editing of Pulsatilla patens mitogenome 


\begin{tabular}{|c|c|c|c|c|c|c|c|}
\hline Category & Gene & Location & $\begin{array}{l}\text { Gene } \\
\text { structure }\end{array}$ & $\begin{array}{l}\text { RNA } \\
\text { editing } \\
\text { events }\end{array}$ & $\begin{array}{l}\text { RNA editing } \\
\text { frequency }\end{array}$ & $\begin{array}{l}\text { CDS } \\
\text { Length } \\
\text { nc/aa }\end{array}$ & $\begin{array}{l}\text { GC } \\
\text { content }\end{array}$ \\
\hline \multirow[t]{9}{*}{ Complex 1} & nad1 & $\begin{array}{l}\text { M1 (e1);M2(e2- } \\
\text { 3);M3(e4-5) }\end{array}$ & $\begin{array}{l}\text { e1;e2-i1- } \\
\text { e3;e4-i2- } \\
\text { e5 }\end{array}$ & 45 & 3,578732106 & 978 & 43,6 \\
\hline & nad2 & $\begin{array}{l}\text { M1(E1-E2); } \\
\text { M3(E3-5) }\end{array}$ & $\begin{array}{l}\text { e1-i1- } \\
\text { e2;e3-i2- } \\
\text { e4-i3-e5 }\end{array}$ & 43 & 2,810457516 & 1530 & 41 \\
\hline & nad3 & ChM3 & continous & 19 & 5,322128852 & 357 & 42 \\
\hline & nad4 & ChM1 & $\begin{array}{l}\text { e1-i1-e2- } \\
\text { i2-e3-i3- } \\
\text { e4 }\end{array}$ & $\begin{array}{l}65 \mathrm{CDS} \\
+3 \\
\text { introns }\end{array}$ & 4,36827957 & 1488 & 42,7 \\
\hline & nad4L & ChM1 & continous & 12 & 4,395604396 & 273 & 37,4 \\
\hline & nad5 & $\begin{array}{l}\text { ChM1 (E1-2), } \\
\text { CHM2 (E3-E4) }\end{array}$ & $\begin{array}{l}\text { e1-i1- } \\
\text { e2,e3-i2- } \\
\text { e4 }\end{array}$ & 39 & 1,937406855 & 2013 & 41,8 \\
\hline & nad6 & ChM1 & continous & 17 & 2,791461412 & 609 & 41,2 \\
\hline & nad7 & ChM2 & $\begin{array}{l}\text { e1-i1-e2- } \\
\text { i2-e3-i3- } \\
\text { e4-i4-e5 }\end{array}$ & $\begin{array}{l}40 \mathrm{CDS} \\
+6 \\
\text { introns }\end{array}$ & 3,410059676 & 1174 & 44,6 \\
\hline & nad9 & ChM2 & continous & 11 & 1,919720768 & 573 & 42,9 \\
\hline Complex 2 & sdh4 & ChM1 & continous & 8 & 1,93236715 & 414 & 38,9 \\
\hline Complex 3 & cob & ChM2 & continous & 22 & 1,842546064 & 1194 & 42,5 \\
\hline \multirow[t]{3}{*}{ Complex 4} & $\operatorname{cox} 1$ & ChM1 & continous & 30 & 1,890359168 & 1587 & 44 \\
\hline & $\operatorname{cox} 2$ & ChM1 & e-i-e & $\begin{array}{l}18- \\
\text { cds, } 1 \\
\text { intron }\end{array}$ & 0,5323868678 & 3381 & 49,4 \\
\hline & $\operatorname{cox} 3$ & ChM1 & continous & 20 & 2,506265664 & 798 & 45,2 \\
\hline \multirow[t]{5}{*}{ Complex 5} & atp1 & ChM1 & continous & 3 & 0,1937984496 & 1548 & 44,3 \\
\hline & atp4 & ChM1 & continous & 12 & 2,116402116 & 567 & 42,5 \\
\hline & atp6 & ChM1 & continous & 25 & 3,267973856 & 765 & 38,7 \\
\hline & atp8 & ChM1 & continous & 9 & 1,851851852 & 486 & 40.1 \\
\hline & atp9 & ChM1 & continous & 1 & 0,4444444444 & 225 & 37,3 \\
\hline \multirow{4}{*}{$\begin{array}{l}\text { Cytochrom c } \\
\text { biogenesis }\end{array}$} & $\mathrm{ccmC}$ & ChM1 & continous & 40 & 5,376344086 & 744 & 44,4 \\
\hline & $\mathrm{ccmB}$ & ChM1 & continous & 42 & 6,763285024 & 621 & 42,7 \\
\hline & $\mathrm{ccmFC}$ & ChM1 & e1-i-e2 & 25 & 1,081782778 & 2311 & 49,7 \\
\hline & ccmFN & ChM1 & continous & 34 & 1,960784314 & 1734 & 47,3 \\
\hline
\end{tabular}




\begin{tabular}{|c|c|c|c|c|c|c|c|}
\hline \multirow{4}{*}{$\begin{array}{l}\text { Ribosome } \\
\text { large subunit }\end{array}$} & rpl2 & ChM1 & e1-i-e2 & 1 & 0,06600660066 & 1515 & 51 \\
\hline & rpl5 & ChM1 & continous & 10 & 1,792114695 & 558 & 44,1 \\
\hline & rpl10 & ChM1 & continous & 6 & 1,19760479 & 501 & 42,9 \\
\hline & rpl16 & ChM1 & continous & 10 & 2,096436059 & 477 & 45,3 \\
\hline \multirow{10}{*}{$\begin{array}{l}\text { Ribosome } \\
\text { small subunit }\end{array}$} & rps1 & ChM1 & continous & 5 & 0,7278020378 & 687 & 42,8 \\
\hline & rps2 & ChM1 & continous & 10 & 1,481481481 & 675 & 38,2 \\
\hline & rps3 & ChM1 & e1-i-e2 & 16 & 0,9523809524 & 1680 & 43,2 \\
\hline & rps4 & ChM3 & continous & 31 & 2,425665102 & 1278 & 39,3 \\
\hline & rps7 & ChM1 & continous & 3 & 0,6711409396 & 447 & 43 \\
\hline & rps11 & ChM1 & continous & 4 & 0,8888888889 & 450 & 44,2 \\
\hline & rps12 & ChM1,ChM3 & continous & 11 & 2,91005291 & 378 & 45,2 \\
\hline & rps13 & ChM2 & continous & 6 & 1,709401709 & 351 & 39,9 \\
\hline & rps14 & ChM2 & continous & 2 & 0,7575757576 & 262 & 40,2 \\
\hline & rps19 & ChM1 & continous & 4 & 1,388888889 & 288 & 39,6 \\
\hline $\begin{array}{l}\text { Translocation } \\
\text { pathway }\end{array}$ & $\mathrm{mttB}$ & ChM1 & continous & 43 & 5,449936629 & 789 & 44,7 \\
\hline Maturases & matR & ChM3 & continous & 19 & 0,9639776763 & 1971 & 52,5 \\
\hline \multirow[t]{3}{*}{ HGT } & DNA pol & chm1 & continous & 0 & 0 & 2850 & 38,7 \\
\hline & $\begin{array}{l}\text { fasciclin- } \\
\text { like }\end{array}$ & chm3 & continous & 0 & 0 & 396 & 50,8 \\
\hline & RNA pol & $\mathrm{CHM} 1$ & continous & 0 & 0 & 2154 & 33,6 \\
\hline
\end{tabular}

Blasting longer orfs ( $>300 \mathrm{bp}$ ) also revealed other genes that are seldom or non-reported from mitogenomes of angiosperms. The $396 \mathrm{bp}$ long orf from chMt3 chromosome was identified as fasciclin-like arabinogalactan protein 21 with the greatest similarity to sequences found in genomes of Medicago truncata (99\% query coverage, 96.2 percent identity) and species of genus Arachis (97\% query coverage and 83-85.4\% percent identity). Unannotated gene was also found in the three chromosomes of Cicer arietinum (100\% QC, $98.7 \mathrm{PI})$. However, all blastx (and blastn in case of Cicer) hits refer to nuclear genomes of Fabales species, suggesting that this gene was recently acquired by intracellular HTG from nucleus or was previously unannotated in known mitogenomes. Blasting against known mitochondrial genomes revealed the presence of this gene $(97-100 \%$ query coverage and $99-95 \%$ percent of identity) not only in mitogenomes of Fabales (Lotus japonicus, Tamarindus indica, Styphnolobium japonicum and Medicago truncata), but also in Paraprenanthes diversifolia and Lactuca species of Asterales. The phylogenetically scattered presence of this gene in angiosperm mitogenomes supports nuclear origin and its transfer to mitogenome independently in at least three angiosperm orders: Fabales, Asterales and reported for the first time Ranunculales. 
The chromosome chMt1 of mitochondrial genome of Pulsatilla patens contains orf encoding RNA-dependent RNA polymerase (RdRP) and an expression of this gene was confirmed by RNA-seq analysis. The RdRp gene is recognized by its conserved protein domain family, pfam05919 and is presumably required for replication of mitoviruses [35] and was found in 40 out of 50 analysed plant mitogenomes [36]. Mitoviruses have small, nonsegmented RNA genomes and all are through to replicate in the host mitochondria [37, 38]. They were previously known from fungal hosts [39] and their detection in plant transcriptomes was considered as a contamination from fungal pathogens or as a result of HGT [36]. However, the recent studies suggest an existence of the contemporary plant mitoviruses [35]. The completeness of RdRP gene in plant mitochondrial genomes varies from nearly complete versions of the RdRP to remnants barely detectable in sequence searches [36]. The RdRP gene found in the Pulsatilla mitogenome is 292 aa long and belongs to the longest among those found in plants. The further studies on Pulsatilla transcriptomes are required to confirm the presence of a complete mitovirus genome, which can be up to $4.4 \mathrm{kbp}$ long [35].

The 328 aa long orf encoding reverse transcriptase with retrotransposon gag protein domain pfam03732 were found on chromosome chMt3 (blastx up to $90 \%$ of query coverage and $>50 \%$ identity), however blasting against known plant mitogenomes did not provide any hits. The discovery of this gene encouraged us to perform LTR search in the assembled mitogenome, which are often considered as HGT vectors among nuclear and mitochondrial genomes [40]. Several orfs longer than 500 bp remain unidentified, as no significant hits were found in public databases, despite their expression end RNA editing events. However, the genomic resources of early eudicots are poorly explored.

Primary annotation of $r p / 2$ gene did not reveal presence of stop codon at the end of the second exon. In the most angiosperms the stop codon of mitochondrial $r p s 2$ is generated by C->U RNA editing process [41, 42, 43]. However, despite deep reads coverage, the editing required to develop stop codon in $r p / 2$ was not detected even at low frequency. This gene in various angiosperm lineages is known by different intermediate stages of its transfer to the nuclear genome [44]. It has been proposed that part of $r p / 2$, located between the intron and $3^{\prime}$ end of the gene, was transferred to the nuclear genome in the ancestor of core eudicots, most likely preceding the generation of a stop codon TAA. That left mitochondrial $r p / 2$ shortened to form present in most angiosperm mitogenomes [45]. Following fission, the 5 ' part of $r p / 2$ was transferred to the nucleus in Fables, lettuce and probably several other eudicot lineages [46]. The mitochondrial $r p s 2$ of $P$. patens is longer than in core eudicots and corresponds to structure found in the early angiosperms: Magnoliales [47] and Nymphaeales [48]. The lack of variant in the $r p / 2$ and surrounding regions suggests, that process if $r p / 2$ transfer to nuclear genome appear after divergence of Ranunculales, but before divergence of Proteales, since mitochondrial genome of Nelumbo nucifera contains shortened $r p / 2$ known from core eudicots [17].

\section{Phylomitogenomics relationships of Pulsatilla patens}

The phylogenomic analyses based on protein-coding mitochondrial genes resulted in trees with similar topologies (Figure 3, Figure S1) and obtained topologies are congruent with previously published papers [49].

The phylogenetics analysis of 39 protein-coding mitochondrial genes reveals Aconitum, Anemone and Pulsatilla (Ranunculales) as early divergent eudicots with Nelumbo nucifera (Proteales) as it's closest relative (Figure 3). However, the phylogenomics analyses did not resolve Proteales-Ranunculales relationships with a maximal support. Previous studies using mitochondrial dataset [49], resolved Nelumbo as a basal for eudicots, however, it 
naturally did not include any member of Ranunculales. The phylogenetic position of Pulsatilla patens is congruent with plastome datasets, which resolved Ranunculales as an earlier divergent than Proteales [50]. Also, the analysis of 1,594 nuclear loci resolved Ranunculales as an older group than Proteales [51] and provided results congruent with chloroplast and mitochondrial data. The main aim of this analysis was the phylogenetic placement of mitogenomes containing polymerase genes (DNApol, RNApol) which are scattered along the whole tree.

Phylogenetic relationships of mitochondrial DNApol and RNApol genes in many cases did not reflect the phylogenetic position of species (Figures S2-S3), suggesting it's independent acquisition via horizontal gene transfer, which was reported several time in plant mitochondrial genomes [4,52,53]. The sequences of polymerase genes extracted from mitogenomes of close related species usually group together, but in some clades even orders are mixed. The phylogenetic position of $P p \_R N A \_p o l$ gene remained unresolved (Figure S3) and $P p \_D N A \_p o /$ gene was clustered together with genes coming from evolutionary unrelated genera like Theobroma, Actinidia and Prunus (Figure S2).

Repeats

Based on the currently known genomic resources, these large repeats seem to be limited to the genus Pulsatilla. The BLAST against known mitogenomes revealed only presence of very short (up to $400 \mathrm{bp}$ in case of Ligustrum sp.) parts of these repeats in other plant species. Each of three P. patens mitochondrial chromosomes is flanked with the same pair of inverted repeats, which seem to be unique among known mitogenomes. Chromosomes of Solanum tubersoum are also flanked with repeats, however in this case, the repeats were present in only two copies and their length ranged from 1,208 to 11,915 bp [12].

Beside mentioned large repeats, which shape the $P$. patens mitogenome structure only two repeats longer than $1,000 \mathrm{bp}$ were found. The repeats $\mathrm{S} 1$ (1,889 bp) and S2 (1,594 bp) were located at chMt2 and present in two copies. Additional two repeats shared among chromosomes fall within 500-1,000 bp range: repeats S3 (848 bp long) were found at chMt1 and chMt2 and repeat S4 (697 bp) were found at chMt2 and chMt3.

\section{Chloroplast derived regions}

DNA transfer from plastome to mitogenome is well documented in almost all vascular plant lineages [29, 54]. This transfer is usually not limited to a single gene, but in most cases it comprises a cluster of genes, which seems to be conserved among angiosperms [55]. The Pulsatilla patens, which belongs to early divergent angiosperm order Ranunculales, reveals over $35 \mathrm{kbp}$ of plastome derived regions in its mitogenome, called MTPT (plastid-derived mitochondrial DNA), which comprise $3.6 \%$ of total mitogenome length (Table S1). The ptderived DNA is distributed among all the mt-chromosomes: 5, 3, and 1 transfer were found in chMt1, chMt2 and chMt3 respectively. Out of 9 unique cp-mtDNA regions, 5 were transferred from chloroplast inverted repeat regions, including longest, over $19 \mathrm{kbp}$. In total, almost the whole IR region (except gene ycf1) was transferred to mitochondrion in scattered form mainly over chMt1 with one part located on chMt2. The length of the longest MTPT is unusual, since the most previously identified chloroplast derived regions fall within 200-4,000 bp range [56] and the largest, $12.6 \mathrm{kbp}$ long MTPT was found in Zea mays mitogenome [57]. In the other known mitogenome of Ranunculales, Hepatica maxima, the MTPT fragments are up tp $7.1 \mathrm{kbp}$ long, with total length of $16 \mathrm{kbp}$ [16] The presence of an almost complete IR region in P. patens mitogenome suggests that it was derived from single transfer that was subsequently split and partially duplicated by rearrangement. Similar 
scenario was described in Silene conica, where $35 \mathrm{kbp}$ region was transferred in a single event but later, most of this MTPT was removed by a series of deletion events [57]. The mitogenome of $P$. patens contains $26.7 \%$ of plastome (excluding one copy of IR) which falls between Cucumis melo (22.7\%) and Bambusa oldhamii (40.9\%) Due to lack of mitogenomic data on early divergent eudicots it's difficult to infer the evolutionary significance of the amount of transferred cpDNA.

Among MTPT gene clusters identified by Wang et al. [55], three were not found in P. patens mitogenome: atpE$r b c \mathrm{~L}, p s b \mathrm{E}-p s b \mathrm{~F}$ and $y c f 1-t r n \mathrm{~N}$. Another transferred cluster specific for eudicots, $p s a \mathrm{~A}-p s a \mathrm{~B}$ [55] was splitted among chromosomes chMt1 and chMt2 (Table S1). The rpoC1-rpoC2 cluster found at chromosome chMt3 was not specific for Pulsatilla, as blasting it against known plant mitogenomes confirmed its presence in six mitogenomes belonging to different orders: Phoenix dactylifera (Lililes), Sapria himalayana (Malpighiales), Solanum aethopicum (Solanales), Ziziphus jujuba (Rosales), Spondias tuberosa (Sapindales) and Ammopiptanthus mongolicus (Fabales). Recent studies hypothesize that plastome sequences were initially acquired by intracellular gene transfer and then were transferred among plant lineages via mitochondrial horizontal gene transfer [56], however in the case of Pulsatilla mitogenome this scenario in rather unlikely. The longest MTPT (chloroplast transfer 4) contains 11 genes which order is characteristic for all known Pulsatilla chloroplast genomes and intergenic spacer sequences is specific for this genus [32, 33, 58, 59]. Separation of MTPT from plastome reads with the whole genome sequencing approach could be challenging, especially while only short reads sequencing results are available, which could be mapped on both regions making them look like each other. Application of two long reads sequencing platform enabled presence of core mtDNA and MTPT in single reads, which confirmed the mitochondrial origin of MTPT which fall within the range of cPDNA variation at intraspecific level [32] suggesting it's acquisition via intracellular gene transfer.

RNA editing

According to previous studies RNA editing is obligatory for few mitochondrial genes by creating initiation or termination codons [12]. In the Pulsatilla patens mitochondrial genome, the $\mathrm{C}-\mathrm{U} \mathrm{U}$ editing is required to create start codon in nad1 and stop codons in atp6, rps11, ccmFC, and cox2 genes.

The mitochondria of flowering plants usually have 300-500 sites that are subjected to RNA editing [60]. Despite considering only editing events with frequency above 0.5 , the number of affected sites (907) is bigger than in Solanum [12] and Populus [61], where 799 and 355 editing events were detected respectively. High number of editing sites in CDS of $P$. patens mitogenome can not be explained by tissue used for RNA extraction, since leaf (as in case of our study) and root tissues are considered as less frequently edited than flower tissues [62]. The lower number of predicted RNA-editing sites was given for Hepatica maxima [16] due to presence of two genes missing in P. patens ( $r p / 10$ and $s d h 4)$. However, predicting-based estimation of RNA-editing sites usually overstates the amount of RNA-seq verified editing sites [63].

The number of observed edited sites varies from single in rp/2 up to 65 in nad4 (Table 1, Fig. S4). Considering gene category, the highest number of edited base pairs was found in the genes of Complex 1 (nad) and Cytochrome $\mathrm{C}$ biogenesis $(\mathrm{ccm})$, which is congruent with data obtained for Populus [61] as well as for early land plants [63]. Despite deep transcriptome sequencing, the reverse U $\rightarrow C$ editing, which was documented in many early divergent tracheophytes lineages [64], has not been observed at assumed frequency in $P$. patens mitogenome. 
In our study, the high number of editing sites can not be explained by possible false-positive results often observed in many surveys, where DNA and RNA libraries were prepared from different individuals.

Interspecific variation in protein- coding genes

The comparative analyses of $P$. alpina, $P$. patens and $P$. pratensis protein coding genes (CDS) revealed 50 SNPs (43 dN and $7 \mathrm{dS}$ ) and 23 (18 dN and $5 \mathrm{dS}$ ) differentiating $P$. patens from $P$. alpina and $P$. pratensis respectively (Table XS2). Eight out of 38 compared genes did not reveal any interspecific variation (nad1, nad3, nad6, nad9, $c o x 3, c c m C, r p / 2, m t t B$ ) and in the next seven genes only single SNPs were detected (nad4, nad4L, nad5, nad7, cox1, ccmB, rps12, matR). The interspecific comparison revealed that the genes of complexes I-IV are least variable, while the most of interspecific variation is accumulated in the genes of Complex $V$ and genes of small ribosomal subunit. Among the genes of Complex $V$ the lowest variation was found in atp9 (only two synonymous mutations), while in the remaining number of nucleotide substitution among atp genes varied from 7 (atp8) to 12 (atp1).

The genes belonging to other groups revealed low variation, except from ccmFC where 16 substitutions were found.

Most mutations found in protein- coding genes were substitutions, however the indels were also identified in five genes including 2aa indel in atp4, 5aa in ccmFC, 3aa in rps1, 4aa indel in rps3 and the largest, 30aa indel in rps4. With single exception of atp4 (3 bp shorter CDS in P. pratensis than P. patens), the rest indels were found in P. alpina.

The mitochondrial genes are usually considered as slower evolving than chloroplast genes [65, 66], but the comparative analyses at genus level are limited to only a few genera.

The number of mutations of Pulsatilla mitochondrial CDS did not deviate from chloroplast CDS, especially when comparing atp and rps genes. Chloroplasts CDS of atpA, atpB, atpE and atpl revealed 3, 2, 1 and 1 substitutions respectively despite analysing six individuals from three Pulsatilla species [32]. Also the genes of small ribosomal subunit of chloroplast genome were less variable, with only two substitutions in rps11 and rps 15. With exception of the most variable ycf1 (39 substitutions), the numbers of substitutions in the remaining, relatively variable chloroplast CDS, ranged from five to seven [32].

The raw numbers of indels and substitutions could be biased by differences in genes length, therefore the pi nucleotide diversity is often used to estimate relative differences in the variation among genes. The pi values in the case of analysed Pulsatilla species (excluding mentioned above non-variable genes) varied from 0.0003 ( $m a t R$, nad5) to 0.0516 for rps4 (Table S3). Nine of the ten most variable genes comprised genes from Complex $\mathrm{V}$ and small ribosomal subunit (Table S4), which corresponded to the raw numbers of detected SNPS.

The mitochondrial protein- coding genes of Pulsatilla genus revealed relatively high variation in comparison to other vascular plants. Only eight SNPs were found among mitochondrial genes in the genus Larix [66]. On the other hand, the number of SNPs at species level in Silene vulgaris was much higher (144 SNPs), but they mostly concentrated into three genes: atp1, atp6 and $\operatorname{cox} 1$ [10]. However, the genus Silene is known for its extreme mitochondrial diversity, not only at substitution level but also in structure and gene content [10].

Mitochondrial genomes as a resource for phylogenetic and phylogeography studies

Page $14 / 24$ 
The plastomes of Ranunculaceae are structurally variable $[32,68]$, but at the genus level the gene order seems to be conserved $[32,33,58,59]$. Currently the data on sequenced mitogenomes of Ranunculaceae are restricted to three species, with different mitogenome structure, gene content and order. However, based on preliminary mappings of sequenced short-read libraries from Pulsatilla alpina, $P$. vernalis and $P$. pratensis, the selection of regions for phylogenetic and phylogeographic studies on generic level is quite challenging due to common presence of large repeats and MTPTs. Moreover, the linearity of preferred regions should be conserved among species. Eight single copy linear regions of minimum length $10 \mathrm{kbp}$ with shared, identical gene order among analysed species were identified at chromosome chMt1 at 89K-122K, 149K-160K, 168K-179K, 123K-252K, 283K299K, 303K-333K, 377K-399K, 409K-424K. Together $165 \mathrm{kbp}$ of the total $452 \mathrm{kbp}$ (36 \%) of chromosome chMt1 were suitable for low-level phylogenetic and phylogeographic studies. In the case of shorter chromosome chMt2, for regions meeting above criteria was found: 83K-94K, 101K-126K, 214K-276K, 295K-323K, which consist 37\% (126K bp) of total chMt2 length. At chromosome chMt3 only two phylogeny suitable regions were found between $49 \mathrm{~K}-67 \mathrm{~K}$ and $80 \mathrm{~K}-100 \mathrm{~K}$, which consisted $19 \%$ (38k bp) of total chMt3 length. The phylogenetic usage of mitogenomes in plant studies is mainly limited to CDS sequences, due to high evolutionary dynamics of molecule structure. The beyond-CDS mitogenomic phylogenetics is limited mainly to early land plants with a stable structure like liverworts [65] and mosses $[6,69,70]$ and few studies on vascular plant taxa where both CDS an intron sequences were used [71]. The amount of shared mitogenomic clusters among Pulsatilla species seems to be high in comparison to genera like Picea, where only two shared clusters up to 9 kbp were found [26]. Mitochondrial conserved regions seem to have a great potential in phylogenetic studies, despite their taxa specific restrictions. Since their application in low- level phylogenetics is not straightforward like plastid genomes, they can provide better phylogenetic resolution than plastomes [72].

\section{Conclusions}

Mitochondrial genome of early divergent eudicots, Pulsatilla patens revealed multichromosomal structure driven by extraordinarily long, flanking repeats, and shed new light on mitogenomics of early eudicots. The presence of longest, continuous MTPT with structure characteristic for Pulsatilla plastomes suggests its acquisition via intracellular transfer, not via mitochondrial HTG as recently hypothesized. The mitogenome sequences of Anemone, Aconitum and Pulsatilla confirm Ranunculales as earliest divergent eudicots.

\section{Declarations}

Supplementary Materials: The following are available online at www.mdpi.com/xxx/s1, Figure S1: Phylogenetic relationships based on amino acids dataset, Figure S2: Phylogeny on mitochondrial DNA polymerases, Figure S3: Phylogeny of mitochondria RNA polymerases, Figure S4: RNA editing of mitochondrial genes, Table S1: Chloroplast derived regions in mitochondrial DNA of Pulsatilla patens, Table S2: Table S3: Pi diversity of protein coding mitochondrial genes, Table S4: Gene contents of Pulsatilla patens and relatives mitogenomes

Author Contributions: Conceptualization, J.S. and M.Sz.; methodology, J.S., K.M, K.K; software, K.Sz., K.M., J.S; formal analysis, M.Ś, K.K., K.Sz.; resources, M.Sz.; data curation, K.M.; writing-original draft preparation, K.Sz., J.S.; writing-review and editing, K.Sz, K.K., K.M., M.Ś., MSz.; visualization, K.Sz., J.S.; supervision, J.S., K.K., M.Sz.; project administration, K.Sz., M.Sz.; funding acquisition, K.Sz., M.Sz. All authors have read and agreed to the published version of the manuscript. 
Funding: This research was funded by the National Science Centre, Poland, grant number MINIATURA 1 DEC2017/01/X/NZ8/00880 and MINIATURA 3 DEC-2019/03/X/NZ9/01374, and "Development Program of the University of Warmia and Mazury in Olsztyn," co-financed by the European Union under the European Social Fund from the Operational Program Knowledge Education Development. Kamil Szandar is a recipient of a scholarship from the program "Interdisciplinary Doctoral Studies in Biology and Biotechnology" (project number POWR.03.05.00-00-Z310/17), which is funded by the European Social Fund.

Institutional Review Board Statement: Not applicable

Availability of data and materials: The mitogenome sequences are deposited in GenBank with accession numbers MZ420977, MZ420978, MZ420979 for chromosomes MtCh1, MtCh2 and MtChr3 respectively. Raw RNA-seq reads are deposited in SRA archive with their SRA data accessions numbers: SRR10230554, SRR10230555, SRR10230556, SRR10230557.

Acknowledgments: This research was funded by the National Science Centre, Poland, grant number MINIATURA 1 DEC-2017/01/X/NZ8/00880 and MINIATURA 3 DEC-2019/03/X/NZ9/01374.

Ethics approval and consent to participate: Not applicable.

Consent for publication: Not applicable.

Competing interests: The authors declare that they have no competing interests.

\section{References}

1. Liu Y, Medina R, Goffinet B. 350 My of Mitochondrial Genome Stasis in Mosses, an Early Land Plant Lineage. Vol. 31, Molecular Biology and Evolution. 2014. p. 2586-91.

2. Wynn EL, Christensen AC. Repeats of Unusual Size in Plant Mitochondrial Genomes: Identification, Incidence and Evolution. Vol. 9, G3-Genes Genomes Genetics. 2019. p. 549-59.

3. Skippington E, Barkman TJ, Rice DW, Palmer JD. Miniaturized mitogenome of the parasitic plant Viscum scurruloideum is extremely divergent and dynamic and has lost all nad genes. Vol. 112, Proceedings of the National Academy of Sciences of the United States of America. 2015. p. E3515-24.

4. Mower JP, Jain K, Hepburn NJ. The Role of Horizontal Transfer in Shaping the Plant Mitochondrial Genome [Internet]. MarechalDrouard L, editor. Mitochondrial Genome Evolution. London: Academic Press Ltd-Elsevier Science Ltd; 2012. p. 41-69. (Advances in Botanical Research; vol. 63).

5. Wang D, Rousseau-Gueutin M, Timmis JN. Plastid sequences contribute to some plant mitochondrial genes. Mol Biol Evol. 2012 Jul;29(7):1707-11.

6. Sawicki J, Plasek V, Ochyra R, Szczecinska M, Slipiko M, Myszczynski K, et al. Mitogenomic analyses support the recent division of the genus Orthotrichum (Orthotrichaceae, Bryophyta). Sci Rep. 2017 Jun 30;7:4408.

7. Dong S, He Q, Zhang S, Wu H, Goffinet B, Liu Y. The mitochondrial genomes of Bazzania tridens and Riccardia planiflora further confirm conservative evolution of mitogenomes in liverworts. bryo. 2019 May;122(1):130-9. 
8. Kozik A, Rowan BA, Lavelle D, Berke L, Schranz ME, Michelmore RW, et al. The alternative reality of plant mitochondrial DNA: One ring does not rule them all. PLOS Genetics. 2019 Aug 30;15(8):e1008373.

9. Asaf S, Khan AL, Khan AR, Waqas M, Kang S-M, Khan MA, et al. Mitochondrial Genome Analysis of Wild Rice (Oryza minuta) and Its Comparison with Other Related Species. PLOS ONE. 2016 Apr 5;11(4):e0152937.

10. Sloan DB, Alverson AJ, Chuckalovcak JP, Wu M, McCauley DE, Palmer JD, et al. Rapid Evolution of Enormous, Multichromosomal Genomes in Flowering Plant Mitochondria with Exceptionally High Mutation Rates. PLOS Biology. 2012 Jan 17;10(1):e1001241.

11. Myszczynski K, Gorski P, Slipiko M, Sawicki J. Sequencing of organellar genomes of Gymnomitrion concinnatum (Jungermanniales) revealed the first exception in the structure and gene order of evolutionary stable liverworts mitogenomes. BMC Plant Biol. 2018 Dec 3;18:321.

12. Varré J-S, D’Agostino N, Touzet P, Gallina S, Tamburino R, Cantarella C, et al. Complete Sequence, Multichromosomal Architecture and Transcriptome Analysis of the Solanum tuberosum Mitochondrial Genome. International Journal of Molecular Sciences. 2019 Jan;20(19):4788.

13. Sloan DB. One ring to rule them all? Genome sequencing provides new insights into the 'master circle' model of plant mitochondrial DNA structure. New Phytologist. 2013;200(4):978-85.

14. Wang S, Song Q, Li S, Hu Z, Dong G, Song C, et al. Assembly of a Complete Mitogenome of Chrysanthemum nankingense Using Oxford Nanopore Long Reads and the Diversity and Evolution of Asteraceae Mitogenomes. Genes (Basel) [Internet]. 2018 Nov 12 [cited 2021 Mar 23];9(11). Available from: https://www.ncbi.nlm.nih.gov/pmc/articles/PMC6267336/

15. Handa $\mathrm{H}$. Linear plasmids in plant mitochondria: Peaceful coexistences or malicious invasions? Mitochondrion. 2008 Jan 1;8(1):15-25.

16. Park S, Park S. Large-scale phylogenomics reveals ancient introgression in Asian Hepatica and new insights into the origin of the insular endemic Hepatica maxima. Sci Rep [Internet]. 2020 Oct 1 [cited 2021 Mar 23];10. Available from: https://www.ncbi.nIm.nih.gov/pmc/articles/PMC7529770/

17. Gui S, Wu Z, Zhang H, Zheng Y, Zhu Z, Liang D, et al. The mitochondrial genome map of Nelumbo nucifera reveals ancient evolutionary features. Sci Rep [Internet]. 2016 Jul 22 [cited 2021 Mar 23];6. Available from: https://www.ncbi.nlm.nih.gov/pmc/articles/PMC4957087/

18. Liu Y, Xue J-Y, Wang B, Li L, Qiu Y-L. The Mitochondrial Genomes of the Early Land Plants Treubia lacunosa and Anomodon rugelii: Dynamic and Conservative Evolution. PLOS ONE. 2011 Oct 5;6(10):e25836.

19. Grewe F, Viehoever P, Weisshaar B, Knoop V. A trans-splicing group I intron and tRNA-hyperediting in the mitochondrial genome of the lycophyte Isoetes engelmannii. Nucleic Acids Res. 2009 Aug;37(15):5093104.

20. Liu Y, Wang B, Cui P, Li L, Xue J-Y, Yu J, et al. The Mitochondrial Genome of the Lycophyte Huperzia squarrosa: The Most Archaic Form in Vascular Plants. PLOS ONE. 2012 Apr 12;7(4):e35168.

21. Hecht J, Grewe F, Knoop V. Extreme RNA editing in coding islands and abundant microsatellites in repeat sequences of Selaginella moellendorffii mitochondria: the root of frequent plant mtDNA recombination in early tracheophytes. Genome Biol Evol. 2011;3:344-58.

22. Guo W, Zhu A, Fan W, Mower J. Complete mitochondrial genomes from the ferns Ophioglossum californicum and Psilotum nudum are highly repetitive with the largest organellar introns. New Phytologist. 
2016 Aug 1;213.

23. Chaw S-M, Shih AC-C, Wang D, Wu Y-W, Liu S-M, Chou T-Y. The mitochondrial genome of the gymnosperm Cycas taitungensis contains a novel family of short interspersed elements, Bpu sequences, and abundant RNA editing sites. Mol Biol Evol. 2008 Mar;25(3):603-15.

24. Kan S-L, Shen T-T, Gong P, Ran J-H, Wang X-Q. The complete mitochondrial genome of Taxus cuspidata (Taxaceae): eight protein-coding genes have transferred to the nuclear genome. BMC Evol Biol. 2020 Jan 20;20(1):10.

25. Jackman SD, Warren RL, Gibb EA, Vandervalk BP, Mohamadi H, Chu J, et al. Organellar Genomes of White Spruce (Picea glauca): Assembly and Annotation. Genome Biol Evol. 2015 Dec 8;8(1):29-41.

26. Sullivan AR, Eldfjell Y, Schiffthaler B, Delhomme N, Asp T, Hebelstrup KH, et al. The Mitogenome of Norway Spruce and a Reappraisal of Mitochondrial Recombination in Plants. Genome Biol Evol. 2020 Jan 1;12(1):3586-98.

27. Tsujimura M, Kaneko T, Sakamoto T, Kimura S, Shigyo M, Yamagishi H, et al. Multichromosomal structure of the onion mitochondrial genome and a transcript analysis. Mitochondrion. 2019 May 1;46:179-86.

28. Alverson AJ, Wei X, Rice DW, Stern DB, Barry K, Palmer JD. Insights into the Evolution of Mitochondrial Genome Size from Complete Sequences of Citrullus lanatus and Cucurbita pepo (Cucurbitaceae). Mol Biol Evol. 2010 Jun;27(6):1436-48.

29. Kubo T, Nishizawa S, Sugawara A, Itchoda N, Estiati A, Mikami T. The complete nucleotide sequence of the mitochondrial genome of sugar beet (Beta vulgaris L.) reveals a novel gene for tRNA(Cys)(GCA). Nucleic Acids Res. 2000 Jul 1;28(13):2571-6.

30. Goremykin VV, Lockhart PJ, Viola R, Velasco R. The mitochondrial genome of Malus domestica and the import-driven hypothesis of mitochondrial genome expansion in seed plants. Plant J. 2012 Aug;71(4):61526.

31. Qiu Y, Filipenko SJ, Darracq A, Adams KL. Expression of a transferred nuclear gene in a mitochondrial genome. Current Plant Biology. 2014 Aug 1;1:68-72.

32. Szczecińska M, Sawicki J. Genomic Resources of Three Pulsatilla Species Reveal Evolutionary Hotspots, Species-Specific Sites and Variable Plastid Structure in the Family Ranunculaceae. International Journal of Molecular Sciences. 2015 Sep;16(9):22258-79.

33. Szczecińska M, Łazarski G, Bilska K, Sawicki J. The complete plastid genome and nuclear genome markers provide the molecular evidence of the hybrid origin Pulsatilla $x$ hackelii Pohl. Turkish Journal of Botany. 2017 Mar 1;41.

34. Szczecinska M, Sramko G, Wolosz K, Sawicki J. Genetic Diversity and Population Structure of the Rare and Endangered Plant Species Pulsatilla patens (L.) Mill in East Central Europe. PLoS One. 2016 Mar 22;11(3):e0151730.

35. Nibert ML. Mitovirus UGA(Trp) codon usage parallels that of host mitochondria. Virology. 2017 Jul;507:96100.

36. Bruenn JA, Warner BE, Yerramsetty P. Widespread mitovirus sequences in plant genomes. PeerJ. 2015 Apr 9;3:e876.

37. Hillman BI, Cai G. The family narnaviridae: simplest of RNA viruses. Adv Virus Res. 2013;86:149-76. 
38. Cole TE, Hong Y, Brasier CM, Buck KW. Detection of an RNA-dependent RNA polymerase in mitochondria from a mitovirus-infected isolate of the Dutch Elm disease fungus, Ophiostoma novo-ulmi. Virology. 2000 Mar 15;268(2):239-43.

39. Abdoulaye AH, Cheng J, Fu Y, Jiang D, Xie J. Complete genome sequence of a novel mitovirus from the phytopathogenic fungus Rhizoctonia oryzae-sativae. Arch Virol. 2017 May 1;162(5):1409-12.

40. Hou Y, Rajagopal J, Irwin PA, Voytas DF. Retrotransposon vectors for gene delivery in plants. Mobile DNA. 2010 Aug 2;1(1):19.

41. Gray MW, Burger G, Lang BF. Mitochondrial Evolution. Science. 1999 Mar 5;283(5407):1476-81.

42. Grewe F, Herres S, Viehöver P, Polsakiewicz M, Weisshaar B, Knoop V. A unique transcriptome: 1782 positions of RNA editing alter 1406 codon identities in mitochondrial mRNAs of the lycophyte Isoetes engelmannii. Nucleic Acids Res. 2011 Apr;39(7):2890-902.

43. Baev V, Ivanova Z, Yahubyan G, Toneva V, Apostolova E, Minkov G, et al. Analysis of the complete mitochondrial genome sequence of the resurrection plant Haberlea rhodopensis. Acta Biochimica Polonica. 2021 May 12;68(2):277-86.

44. Szafranski P. Intercompartmental Piecewise Gene Transfer. Genes (Basel). 2017 Oct 6;8(10).

45. Adams KL, Ong HC, Palmer JD. Mitochondrial gene transfer in pieces: fission of the ribosomal protein gene rpl2 and partial or complete gene transfer to the nucleus. Mol Biol Evol. 2001 Dec;18(12):2289-97.

46. Adams KL, Palmer JD. Evolution of mitochondrial gene content: gene loss and transfer to the nucleus. Mol Phylogenet Evol. 2003 Dec;29(3):380-95.

47. Richardson A, Rice D, Young G, Alverson A, Palmer J. The 'fossilized' mitochondrial genome of Liriodendron tulipifera: Ancestral gene content and order, ancestral editing sites, and extraordinarily low mutation rate. BMC biology. 2013 Apr 15;11:29.

48. Dong S, Zhao C, Chen F, Liu Y, Zhang S, Wu H, et al. The complete mitochondrial genome of the early flowering plant Nymphaea colorata is highly repetitive with low recombination. BMC Genomics. 2018 Aug 14;19(1):614.

49. Dong S, Chen L, Liu Y, Wang Y, Zhang S, Yang L, et al. The draft mitochondrial genome of Magnolia biondii and mitochondrial phylogenomics of angiosperms. PLOS ONE. 2020 Apr 15;15(4):e0231020.

50. Gitzendanner MA, Soltis PS, Wong GK-S, Ruhfel BR, Soltis DE. Plastid phylogenomic analysis of green plants: A billion years of evolutionary history. Am J Bot. 2018 Mar;105(3):291-301.

51. Yang L, Su D, Chang X, Foster CSP, Sun L, Huang C-H, et al. Phylogenomic Insights into Deep Phylogeny of Angiosperms Based on Broad Nuclear Gene Sampling. Plant Communications. 2020 Mar 9;1(2):100027.

52. Richardson AO, Palmer JD. Horizontal gene transfer in plants. Journal of Experimental Botany. 2007 Jan $1 ; 58(1): 1-9$.

53. Mower JP, Stefanović S, Hao W, Gummow JS, Jain K, Ahmed D, et al. Horizontal acquisition of multiple mitochondrial genes from a parasitic plant followed by gene conversion with host mitochondrial genes. BMC Biology. 2010 Dec 22;8(1):150.

54. Turmel M, Otis C, Lemieux C. The Mitochondrial Genome of Chara vulgaris: Insights into the Mitochondrial DNA Architecture of the Last Common Ancestor of Green Algae and Land Plants. The Plant Cell. 2003 Aug 1;15(8):1888-903. 
55. Wang D, Wu Y-W, Shih AC-C, Wu C-S, Wang Y-N, Chaw S-M. Transfer of Chloroplast Genomic DNA to Mitochondrial Genome Occurred At Least 300 MYA. Molecular Biology and Evolution. 2007 Sep 1;24(9):2040-8.

56. Gandini CL, Sanchez-Puerta MV. Foreign Plastid Sequences in Plant Mitochondria are Frequently Acquired Via Mitochondrion-to-Mitochondrion Horizontal Transfer. Scientific Reports. 2017 Mar 6;7(1):43402.

57. Sloan DB, Wu Z. History of Plastid DNA Insertions Reveals Weak Deletion and AT Mutation Biases in Angiosperm Mitochondrial Genomes. Genome Biol Evol. 2014 Nov 21;6(12):3210-21.

58. Liu H, He J, Ding C, Lyu R, Pei L, Cheng J, et al. Comparative Analysis of Complete Chloroplast Genomes of Anemoclema, Anemone, Pulsatilla, and Hepatica Revealing Structural Variations Among Genera in Tribe Anemoneae (Ranunculaceae). Front Plant Sci [Internet]. 2018 [cited 2021 May 20];9. Available from: https://www.frontiersin.org/articles/10.3389/fpls.2018.01097/full

59. Li Q, Su N, Zhang L, Tong R, Zhang X, Wang J, et al. Chloroplast genomes elucidate diversity, phylogeny, and taxonomy of Pulsatilla (Ranunculaceae). Scientific Reports. 2020 Nov 13;10(1):19781.

60. Liu Y-J, Xiu Z-H, Meeley R, Tan B-C. Empty Pericarp5 Encodes a Pentatricopeptide Repeat Protein That Is Required for Mitochondrial RNA Editing and Seed Development in Maize[W]. Plant Cell. 2013 Mar;25(3):868-83.

61. Brenner WG, Mader M, Müller NA, Hoenicka H, Schroeder H, Zorn I, et al. High Level of Conservation of Mitochondrial RNA Editing Sites Among Four Populus Species. G3 (Bethesda). 2019 Jan 7;9(3):709-17.

62. Wu B, Chen H, Shao J, Zhang H, Wu K, Liu C. Identification of Symmetrical RNA Editing Events in the Mitochondria of Salvia miltiorrhiza by Strand-specific RNA Sequencing. Sci Rep. 2017 Feb 10;7(1):42250.

63. Myszczyński K, Ślipiko M, Sawicki J. Potential of Transcript Editing Across Mitogenomes of Early Land Plants Shows Novel and Familiar Trends. Int J Mol Sci. 2019 Jun 18;20(12).

64. Knie N, Grewe F, Fischer S, Knoop V. Reverse U-to-C editing exceeds C-to-U RNA editing in some ferns - a monilophyte-wide comparison of chloroplast and mitochondrial RNA editing suggests independent evolution of the two processes in both organelles. BMC Evol Biol [Internet]. 2016 Jun 21 [cited 2021 May 20];16. Available from: https://www.ncbi.nlm.nih.gov/pmc/articles/PMC4915041/

65. Myszczyński K, Bączkiewicz A, Buczkowska K, Ślipiko M, Szczecińska M, Sawicki J. The extraordinary variation of the organellar genomes of the Aneura pinguis revealed advanced cryptic speciation of the early land plants. Scientific Reports. 2017 Aug 29;7(1):9804.

66. Liu Y, Johnson MG, Cox CJ, Medina R, Devos N, Vanderpoorten A, et al. Resolution of the ordinal phylogeny of mosses using targeted exons from organellar and nuclear genomes. Nature Communications. 2019 Apr 2;10(1):1485.

67. Zimmermann HH, Harms L, Epp LS, Mewes N, Bernhardt N, Kruse S, et al. Chloroplast and mitochondrial genetic variation of larches at the Siberian tundra-taiga ecotone revealed by de novo assembly. PLOS ONE. 2019 Jul 10;14(7):e0216966.

68. Zhai W, Duan X, Zhang R, Guo C, Li L, xu G, et al. Chloroplast Genomic Data Provide New and Robust Insights into the Phylogeny and Evolution of the Ranunculaceae. Molecular Phylogenetics and Evolution. 2019 Feb 1;135.

69. Shaw AJ, Devos N, Liu Y, Cox CJ, Goffinet B, Flatberg KI, et al. Organellar phylogenomics of an emerging model system: Sphagnum (peatmoss). Annals of Botany. 2016 Aug 1;118(2):185-96. 
70. Sawicki J, Szczecińska M, Bedarek-Ochyra H, Ochyra R. Mitochondrial phylogenomics supports splitting the traditionally conceived genus Racomitrium (Bryophyta: Grimmiaceae). Nova Hedwigia. 2015 Feb 17;100.

71. Fonseca L, Lohmann L. Exploring the potential of nuclear and mitochondrial sequencing data generated through genome-skimming for plant phylogenetics: A case study from a clade of neotropical lianas. Journal of Systematics and Evolution. 2019 Jul 1;58.

72. Van de Paer C, Bouchez O, Besnard G. Prospects on the evolutionary mitogenomics of plants: A case study on the olive family (Oleaceae). Molecular Ecology Resources. 2018 May 1;18:407-23.

73. Ausubel FM. Current protocols in molecular biology. Brooklyn, N.Y.: Media, Pa: Greene Pub. Associates ; J. Wiley, order fulfillment; 1987. 2 p.

74. Doyle JJ, Doyle JL. Isolation of plant DNA from fresh tissue. Focus. 1990;12:13-5.

75. Li H. Fast construction of FM-index for long sequence reads. Bioinformatics. 2014 Nov 15;30(22):3274-5.

76. Wang JR, Holt J, McMillan L, Jones CD. FMLRC: Hybrid long read error correction using an FM-index. BMC Bioinformatics. 2018 Feb 9;19(1):50.

77. Dierckxsens N, Mardulyn P, Smits G. NOVOPlasty: de novo assembly of organelle genomes from whole genome data. Nucleic Acids Research. 2017 Feb 28;45(4):e18-e18.

78. Lagesen K, Hallin P, Rødland EA, Staerfeldt H-H, Rognes T, Ussery DW. RNAmmer: consistent and rapid annotation of ribosomal RNA genes. Nucleic Acids Res. 2007;35(9):3100-8.

79. Lowe TM, Eddy SR. tRNAscan-SE: a program for improved detection of transfer RNA genes in genomic sequence. Nucleic Acids Res. 1997 Mar 1;25(5):955-64.

80. Ou S, Jiang N. LTR_retriever: A Highly Accurate and Sensitive Program for Identification of Long Terminal Repeat Retrotransposons. Plant Physiology. 2018 Feb 1;176(2):1410-22.

81. Wang Z, Lian J, Li Q, Zhang P, Zhou Y, Zhan X, et al. RES-Scanner: a software package for genome-wide identification of RNA-editing sites. GigaScience [Internet]. 2016 Dec 1 [cited 2021 Mar 23];5(s13742-0160143-4). Available from: https://doi.org/10.1186/s13742-016-0143-4

82. Katoh K, Standley DM. MAFFT multiple sequence alignment software version 7: improvements in performance and usability. Mol Biol Evol. 2013 Apr;30(4):772-80.

83. Nguyen L-T, Schmidt HA, von Haeseler A, Minh BQ. IQ-TREE: A Fast and Effective Stochastic Algorithm for Estimating Maximum-Likelihood Phylogenies. Molecular Biology and Evolution. 2015 Jan 1;32(1):268-74.

84. Minh BQ, Nguyen MAT, von Haeseler A. Ultrafast approximation for phylogenetic bootstrap. Mol Biol Evol. 2013 May;30(5):1188-95.

85. Guindon S, Dufayard J-F, Lefort V, Anisimova M, Hordijk W, Gascuel O. New Algorithms and Methods to Estimate Maximum-Likelihood Phylogenies: Assessing the Performance of PhyML 3.0. Systematic Biology. 2010 May 1;59(3):307-21.

86. Tavare S. Some Probabilistic and Statistical Problems in the Analysis of DNA Sequences. Lectures on Mathematics in the Life Sciences. 1986;17:57-86.

87. Gu X, Fu YX, Li WH. Maximum likelihood estimation of the heterogeneity of substitution rate among nucleotide sites. Molecular Biology and Evolution. 1995 Jul 1;12(4):546-57.

88. Bradbury PJ, Zhang Z, Kroon DE, Casstevens TM, Ramdoss Y, Buckler ES. TASSEL: software for association mapping of complex traits in diverse samples. Bioinformatics. 2007 Oct 1;23(19):2633-5. 


\section{Mitochondrial genome of Pulsatilla patens - 986,613 bp}

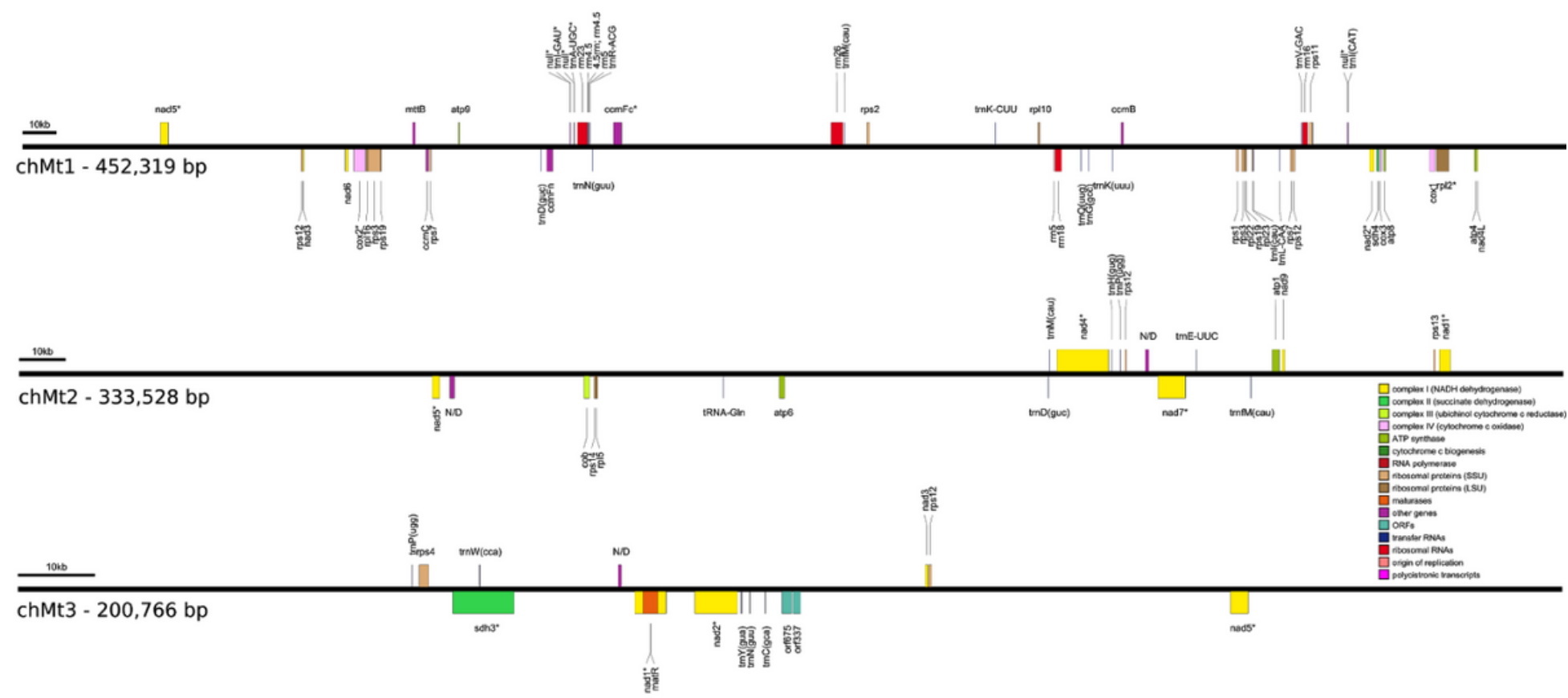

chMt1

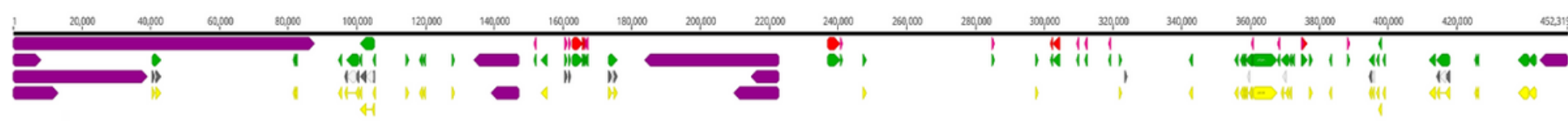

chMt2

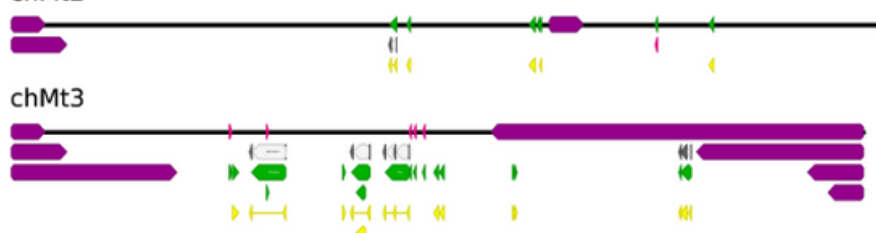

Figure 1

The mitogenome map of Pulsatilla patens. The mitochondrial gene order and chromosomal localization are given in the upper part of the draw - the chromosomes are rescaled. The lower part presents all genes, including MTPTs and flanking repeats.

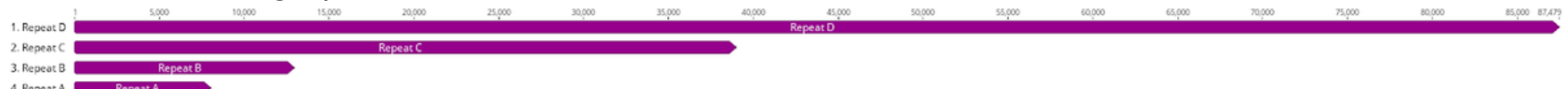

3. Repeat B Repear B

4. Repeat A Repeath

\section{Figure 2}

The alignment of chromosome flanking repeats. 


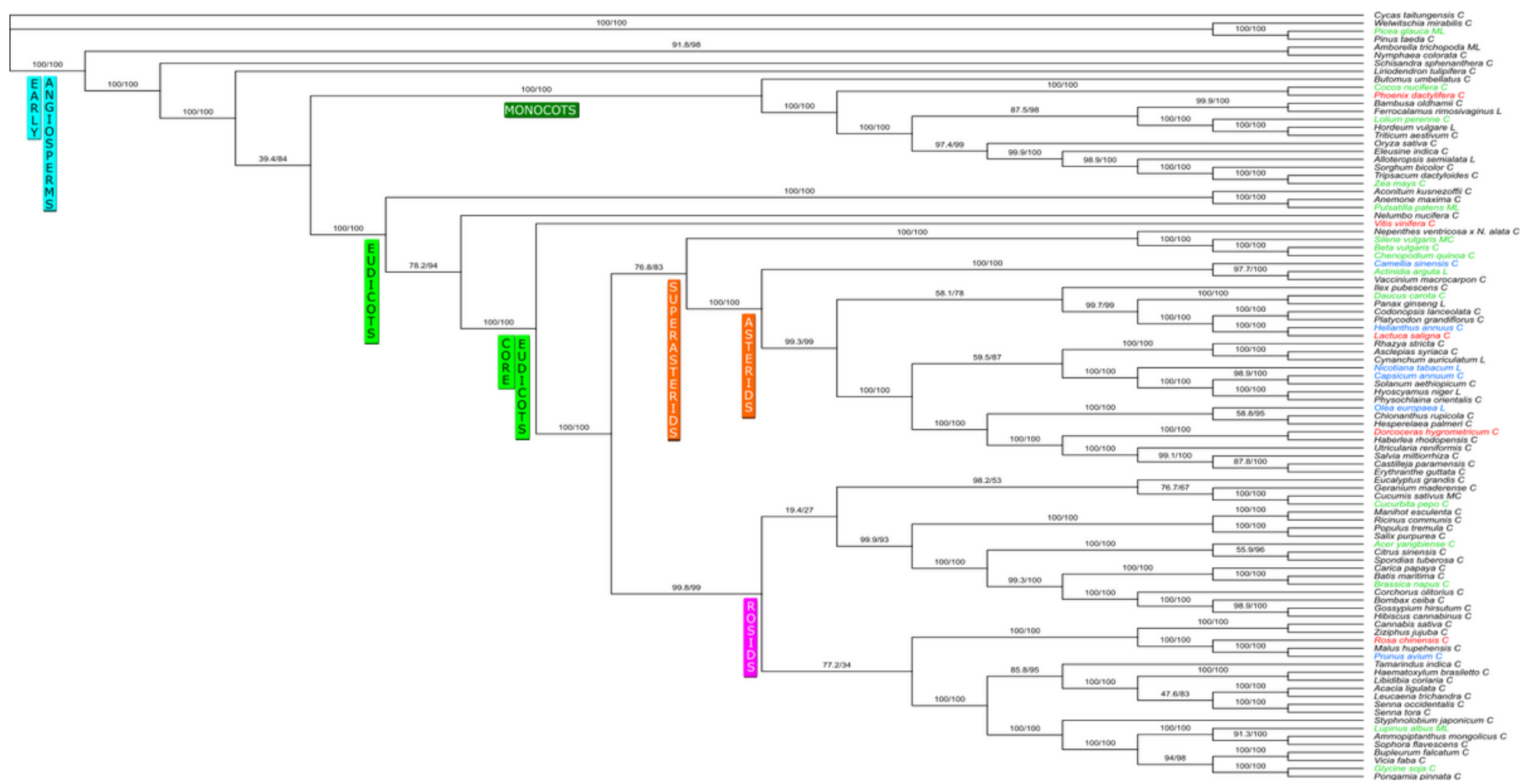

\section{Figure 3}

Phylogenetic tree based on protein-coding mitochondrial genes (green species names - genus contain both polymerase genes (DNApol, RNApol), blue species names - genus contain only DNA polymerase genes (DNApol), red species names - genus contain only RNA polymerase genes (RNApol); C - circular mitogenome, L - linear mitogenome, MC - multicircular mitogenome, ML - multilinear mitogenome). 


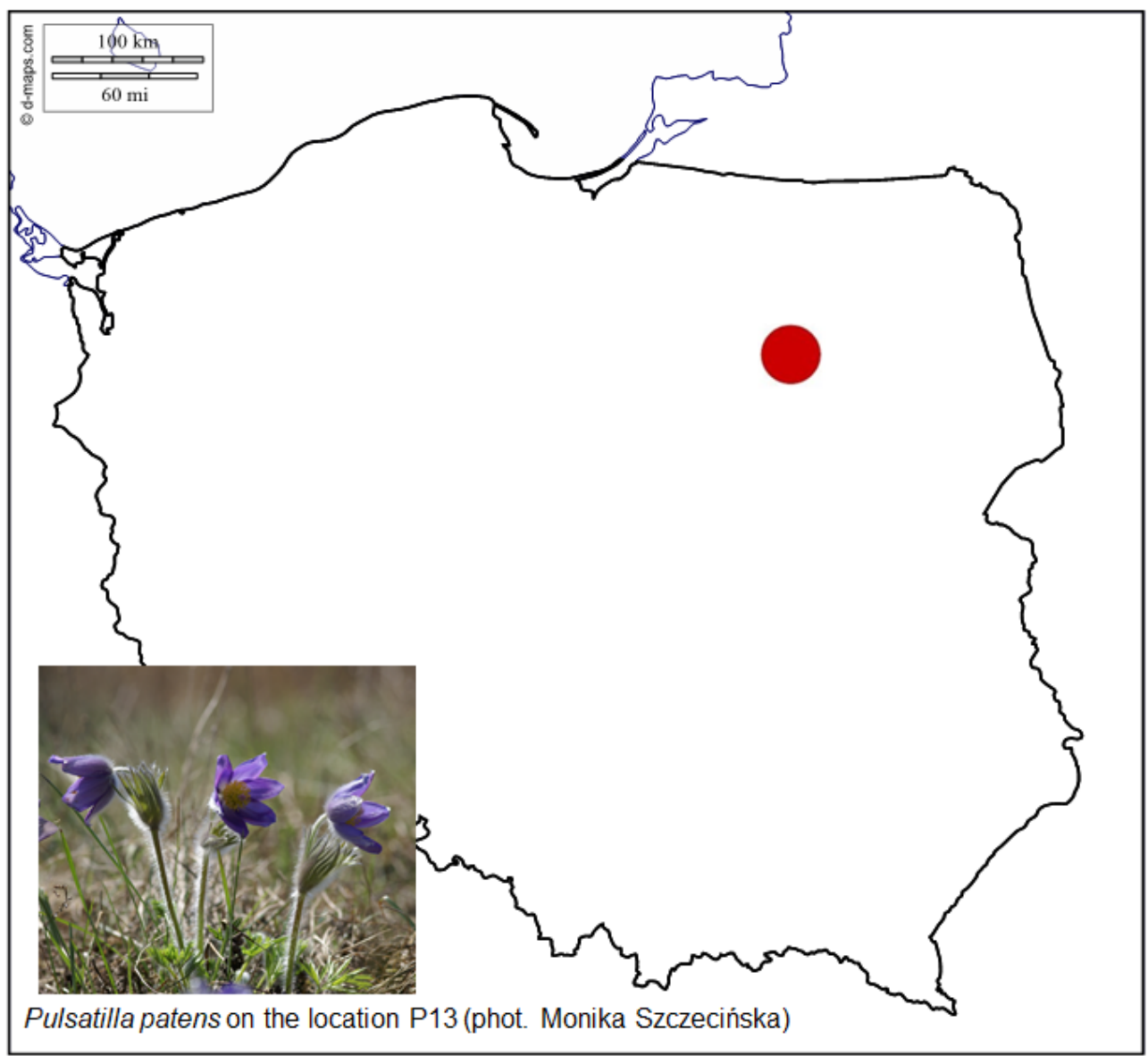

Figure 4

Location of sampled specimens of Pulsatilla patens.

\section{Supplementary Files}

This is a list of supplementary files associated with this preprint. Click to download.

- Tablesuplementary.docx

- FigureS1AA.png

- FigureS2DNA.png

- FigureS3RNA.png

- FigureS4RNAediting.png 